\title{
Factores determinantes en el éxito de un tratamiento antitabaco
}

\author{
Natalia Tamblay ${ }^{1}$ D aniel Seijas ${ }^{2}$. \\ Factors associated with abstinence \\ in a smoking cessation program
}

Background: Smoking cessation programs have an efficacy of approximately 30\%. Different factors related to the patients may influence this figure. Aim: To identify determinant factors for smoking cessation after one year of treatment and to determine if bupropion and nicotine substitutes are effective in smoking cessation treatments. Material and methods: Follow up of 68 patients that attended a smokers clinic at a General Hospital. The patients filled up a questionnaire which included demographic, morbid and smoking habits data. They were subjected to a psychiatric interview to determine their treatment. One year later, patients were contacted by telephone and were asked if they remained without smoking. Results: After one year, $41 \%$ of patients responded that they were abstinent. On univariate analysis, male gender appeared as a protective factor associated to abstinence. On multivariate analysis, the use of bupropion appeared as a protective factor. A high score on the automatic item of the smoking motivation questionnaire appeared as a risk factor. The presence of respiratory diseases and the male gender were borderline significant protective factors. Nicotine substitutes were not associated with better abstinence rates. Conclusions: In this sample of smokers, the use of bupropion was associated with better abstinence rates and a high motivation to smoke appeared as a risk factor to continue smoking (Rev Méd Chile 2008; 136: 179-85). (Key words: Bupropion; Nicotine; Smoking)

Recibido el 4 de abril, 2007. Aceptado el 28 de septiembre, 2007.

${ }^{1}$ Servicio de Salud Metropolitano Central. ${ }^{2}$ Centro de Adicciones, Pontificia Universidad Católica de Chile, Santiago de Chile.

$\mathrm{E}^{\mathrm{n}}$ n el año 2000, en el mundo, uno de cada tres adultos fumaba, lo que equivale a 1.100 millones de personas, si se continúa con la tendencia, este número aumentará a 1.600 millones de fumadores para el año $2025^{1}$.

En Chile, según la encuesta nacional de salud 2003, dentro de los mayores de 18 años, fuman $48 \%$ de las mujeres y $37 \%$ de los hombres ${ }^{2}$. En 2002, el número de muertes atribuibles al tabaco

Correspondencia a: Dra Natalia Tamblay. Victoria Subercaseux 381. Fono: (56 2) 3952256. Fax: (56 2) 4940367. E mail: natalia.tamblay@redsalud.gov.cl

Ubicación actual Dr. Daniel Seijas: Director, Centroeduca, Clínica Las Condes. fue de 13.882, lo que correspondió a $17 \%$ de las muertes totales ${ }^{3}$.

La efectividad del bupropión y los sustitutos de nicotina ( $\mathrm{SN}$ ) se encuentra avalada por la literatura internacional ${ }^{4,5}$. En Chile sólo se ha estudiado la efectividad de los $\mathrm{SN}^{6}$, a nivel nacional se carece de estudios que aborden la efectividad del bupropión y los factores determinantes en el éxito de tratamiento antitabaco.

En este estudio se realizó un seguimiento a 68 pacientes tratados en la Clínica del Fumador de la Pontificia Universidad Católica de Chile, con el propósito de:

a) Identificar los factores determinantes en el cese de fumar a un año de tratamiento, de manera 
de saber, quiénes dentro de la población estudiada se benefician más con un tratamiento de este tipo. b) Determinar si el bupropión y los SN son efectivos dentro de un tratamiento antitabaco, lo que puede orientar en la indicación de estos medicamentos.

\section{MATERIAL Y MÉTODO}

El diseño de este estudio es de cohorte, los sujetos estudiados fueron los pacientes que acudieron a la Clínica del Fumador de la Pontificia Universidad Católica de Chile entre los años 1999 y 2003, con la intención declarada de dejar de fumar y que como parte de su tratamiento asistieron a más de dos sesiones individuales con psiquiatra 0 a terapia grupal (los grupos estuvieron formados por 6 a 10 sujetos, quienes participaron en 10 sesiones junto a dos terapeutas, un psiquiatra y una enfermera), se excluyó a los pacientes con un cuadro psiquiátrico concomitante como trastorno de ánimo y ansiedad en fase aguda o dependencia a alcohol o a drogas ilegales; quedando un total de 105 pacientes, fueron excluidos luego todos aquellos pacientes que no se pudieron ubicar y un paciente que se negó a responder el instrumento. Por todo esto la muestra se redujo a 68 pacientes lo que corresponde a $64,76 \%$ de la muestra inicial.

A aquellos pacientes que ingresaron al estudio, se les entregó un formulario para ser llenado por ellos y el terapeuta, se les consultó si aceptaban llenarlo, previa explicación de la investigación. Hubo un paciente que rechazó llenar el formulario y recibió tratamiento de igual modo que aquellos que aceptaron ingresar al estudio, los datos que incluye el formulario son:

Demográficos: edad y género. Mórbidos: presencia de patología respiratoria, presencia de hipertensión arterial (HTA), antecedente de depresión, consumo de benzodiazepinas, cuestionario de Goldberg abreviado ${ }^{7}$ e índice de masa corporal (IMC). De hábito tabáquico: presencia de algún tabáquico en el hogar, consumo diario de cigarrillos, percepción de autoeficacia, percepción de motivación, puntaje en los ítems del cuestionario de motivación para fumar de la National Addiction Center Smoker Clinic Institute of Psychiatry London University y puntaje en el cuestionario de
Fagerstrom ${ }^{8}$. Tratamiento: uso de bupropión y uso de SN.

$\mathrm{Al}$ inicio, se realizó una entrevista psiquiátrica, orientada a identificar cuadros psiquiátricos concomitantes. Los pacientes siguieron un tratamiento individual o grupal según criterios clínicos, que incluyó en algunos pacientes tratamiento farmacológico. La indicación de bupropión fue por 8 semanas y de los $\mathrm{SN}$, mientras persistieran los síntomas de abstinencia.

Se localizó telefónicamente a los pacientes, y se les preguntó acerca de la abstinencia un año después del inicio del tratamiento y el uso de fármacos; este último dato se corroboró con la información contenida en las fichas clínicas.

Estadística. Los datos fueron ingresados en una planilla Excel, el análisis estadístico se hizo mediante el paquete computacional STATA 7.0. Se obtuvo indicadores de resumen para cada variable, de modo de describir la muestra. Se realizó luego un análisis de regresión logística univariada entre las distintas variables independientes y la variable dependiente y un análisis de regresión logística multivariada a través del método stepwise, con el fin de obtener un modelo final que determine las variables predictoras. Se consideró significativo un valor de P menor a 0,05.

\section{RESUlTADos}

a) Caracterización de la muestra. El 66,18\% de la muestra no presentó patología respiratoria y 33,82\% sí (Tabla 1). El porcentaje de pacientes con hipertensión arterial (HTA) se observa en la Tabla 1 (27,94\% presentó HTA y 72,06\% no).

En relación a la salud mental, 63,08\% de los pacientes tuvo el antecedente de depresión y $36,92 \%$ no (Tabla 1), en relación al consumo de benzodiazepinas 30,88\% de los pacientes fue usuario de éstas al momento del ingreso al tratamiento. La variable consumo diario de cigarrillos previo al tratamiento presentó una mediana de 20 cigarrillos.

Del total de pacientes que ingresaron a tratamiento, 58,21\% recibió bupropión y 41,79\% no (Tabla 1), diferencia que no es significativa. Al $77,94 \%$ de los pacientes se le indicó SN y a $22,06 \%$ no, esta diferencia sí resultó significativa estadísticamente. Cuando se evaluó si el grupo que recibió 
Tabla 1. C aracterísticas de la muestra agrupada por género

\begin{tabular}{|c|c|c|c|c|}
\hline Características & $\begin{array}{l}\text { Total } \\
\mathrm{N}=68\end{array}$ & $\begin{array}{c}\text { Hombres } \\
\mathrm{N}=37\end{array}$ & $\begin{array}{l}\text { Mujeres } \\
\mathrm{N}=31\end{array}$ & $\begin{array}{c}\text { P value } \\
\text { (diferencia } \\
\text { entre géneros) }\end{array}$ \\
\hline \multicolumn{5}{|l|}{ Demográficas } \\
\hline Género femenino & $45,59 \%$ & - & & - \\
\hline Edad (años) & $46,64 \pm 10,7$ & $45,94 \pm 10,93$ & $47,25 \pm 10,61$ & 0,62 \\
\hline \multicolumn{5}{|l|}{ Mórbidas } \\
\hline Presencia patología respiratoria & $33,82 \%$ & $37,8 \%$ & $29 \%$ & 0,44 \\
\hline Presencia HTA & $27,94 \%$ & $27,0 \%$ & $29 \%$ & 0,85 \\
\hline Antecedente de depresión & $63,08 \%$ & $64,7 \%$ & $61,3 \%$ & 0,78 \\
\hline Uso de benzodiazepinas & $30,88 \%$ & $32,4 \%$ & $29 \%$ & 0,76 \\
\hline P. Goldberg $\geq 5$ & $23,53 \%$ & $21,6 \%$ & $25,8 \%$ & 0,69 \\
\hline IMC & $25,34 \pm 4,03$ & $27,23 \pm 3,16$ & $22,91 \pm 3,75$ & 0,0000 \\
\hline \multicolumn{5}{|l|}{ Hábito tabáquico } \\
\hline Presencia fumador en el hogar & $47,76 \%$ & $48,6 \%$ & $46,7 \%$ & 0,87 \\
\hline Consumo promedio diario (mediana) & 20 & 20 & 20 & 0,78 \\
\hline P. autoeficacia $\leq 7$ & $33,33 \%$ & $72,2 \%$ & $59,3 \%$ & 0,28 \\
\hline P. automotivación $\leq 7$ & $16,92 \%$ & $86,5 \%$ & $78,6 \%$ & 0,4 \\
\hline \multicolumn{5}{|l|}{ P. motivación para fumar } \\
\hline Item Act. mano $\geq 5$ & $20,59 \%$ & $21,62 \%$ & $19,35 \%$ & 0,82 \\
\hline Item indulgencia $\geq 5$ & $48,53 \%$ & $40,54 \%$ & $58,06 \%$ & 0,15 \\
\hline Item sedación $\geq 5$ & $63,24 \%$ & $56,76 \%$ & $70,97 \%$ & 0,23 \\
\hline Item estimulación $\geq 5$ & $44,12 \%$ & $37,84 \%$ & $51,61 \%$ & 0,25 \\
\hline Item adicción $\geq 5$ & $79,41 \%$ & $75,68 \%$ & $83,87 \%$ & 0,41 \\
\hline Item automático $\geq 5$ & $22,06 \%$ & $18,9 \%$ & $25,8 \%$ & 0,5 \\
\hline P. Fagerstrom $\geq 7$ & $48,53 \%$ & $43,2 \%$ & $54,8 \%$ & 0,34 \\
\hline \multicolumn{5}{|l|}{ Tratamiento } \\
\hline Uso de Bupropión & $58,21 \%$ & $64,86 \%$ & $50 \%$ & 0,23 \\
\hline Uso de sustitutos de nicotina & $77,94 \%$ & $75,68 \%$ & $80,65 \%$ & 0,62 \\
\hline
\end{tabular}

HTA: hipertensión arterial

IMC: índice de masa corporal

alguno de estos tratamientos era diferente al que no recibió no se encontraron diferencias significativas, salvo para la variable ítem automático de la escala de motivación para fumar. La proporción de los que recibieron bupropión fue mayor en aquellos con un puntaje mayor o igual a 5 en el ítem automático, comparado con aquellos con un puntaje menor de 5 .

Del total de pacientes que ingresaron a tratamiento, 41,18\% (CI 29,18 -53,18) permaneció abstinente al año de tratamiento y 58,82\% estaba fumando luego de un año de tratamiento (Figura 1).

b) Determinantes en la persistencia de fumar al año de seguimiento. Al realizar una regresión logística univariada con todas las variables en estudio, aparece como factor de riesgo de la abstinencia a un año sólo el género femenino (95\% IC 1,2-9,5 OR =3,38). Cuando se ajustan por las otras variables en estudio, mediante regresión logística multivariada, resultaron significativos como determinantes de abstinencia a un año de tratamiento: el bupropión, comportándose como factor protector (95\% IC 0,74-0,86 OR $=0,25)$ y el puntaje alto en el ítem automático del cuestionario de motivación para fumar (95\% IC 1,28-41,6 OR =7,3). El intervalo de confianza para el bupropión es estrecho por tanto el OR tiene alta validez (Tabla 2) (Figuras 2 y 3). 


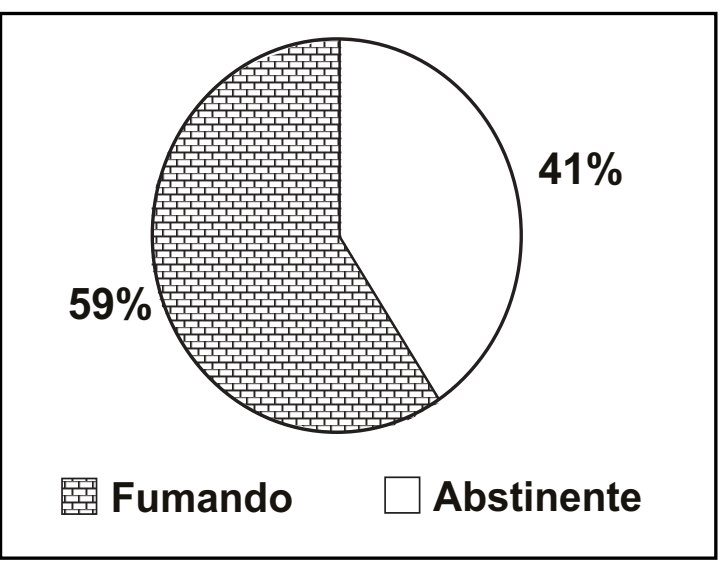

Figura 1. Porcentaje abstinencia de la muestra.

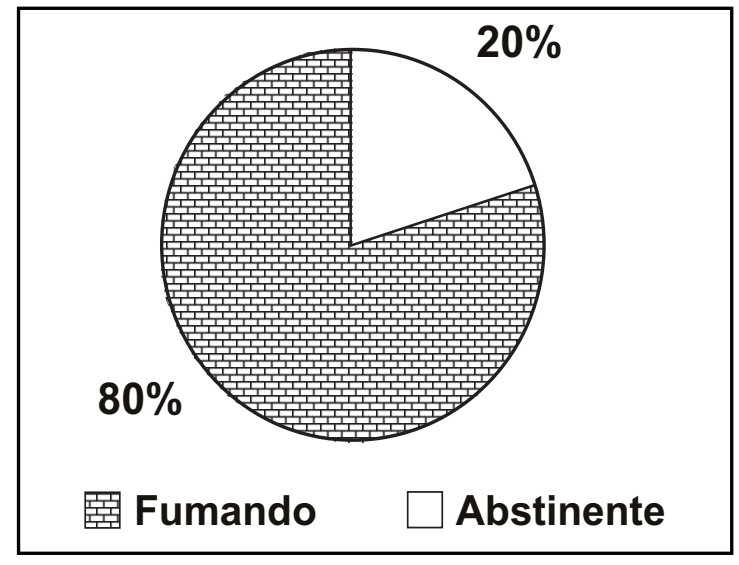

Figura 2. Porcentaje de abstinencia en el grupo con item automático $\geq 5$.

Tabla 2. Predictores de abstinencia al año de tratamiento

\begin{tabular}{|c|c|c|}
\hline Características & $\begin{array}{l}\text { Valor de p regresión } \\
\text { univariada }\end{array}$ & $\begin{array}{l}\text { Valor de p regresión } \\
\text { multivariada }\end{array}$ \\
\hline Género masculino & $\begin{array}{c}\text { OR =0,3 (95\% IC 0,11-0,83) } \\
\text { (valor de p: } 0,02)\end{array}$ & ns (valor de p: 0,07) \\
\hline Edad & ns & ns \\
\hline Presencia patología respiratoria & ns & ns (valor de p: 0,06) \\
\hline Presencia HTA & ns & ns \\
\hline Antecedente de depresión & ns & ns \\
\hline Uso de benzodiazepinas & ns & ns \\
\hline P. Goldberg $\geq 5$ & ns & ns \\
\hline IMC & ns & ns \\
\hline Presencia fumador en el hogar & ns & ns \\
\hline Consumo promedio diario & ns & ns \\
\hline P. autoeficacia $\leq 7$ & ns & ns \\
\hline P. automotivación $\leq 7$ & ns & ns \\
\hline P. motivación para fumar & ns & ns \\
\hline Item act. mano $\geq 5$ & ns & ns \\
\hline Item indulgencia $\geq 5$ & ns & ns \\
\hline Item sedación $\geq 5$ & ns & ns \\
\hline Item estimulación $\geq 5$ & ns & ns \\
\hline Item adicción $\geq 5$ & ns & ns \\
\hline Item automático $\geq 5$ & ns & $\begin{array}{c}\text { OR }=7,3 \text { (95\%IC 1,28-41,6) } \\
\text { (valor de p: } 0,03 \text { ) }\end{array}$ \\
\hline P. Fagerstrom $\geq 7$ & ns & ns \\
\hline Uso de Bupropión & ns & $\begin{aligned} \text { OR }= & 0,25(95 \% \text { IC } 0,74-0,86) \\
& \text { (valor de p: } 0,03)\end{aligned}$ \\
\hline Uso de sustitutos de nicotina & ns & ns \\
\hline
\end{tabular}

ns: no significativo 


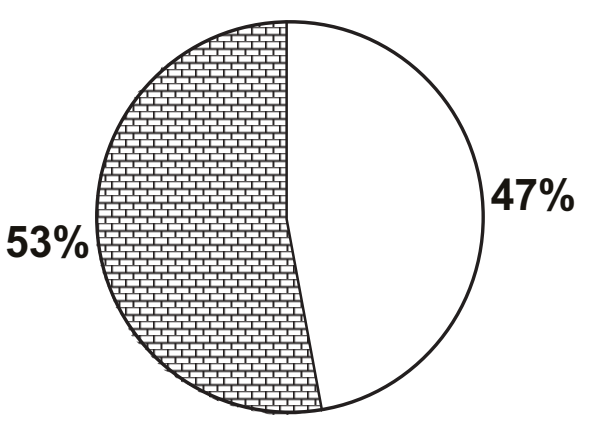

Fumando

Figura 3. Porcentaje de abstinencia en el grupo con item automático $<4$.

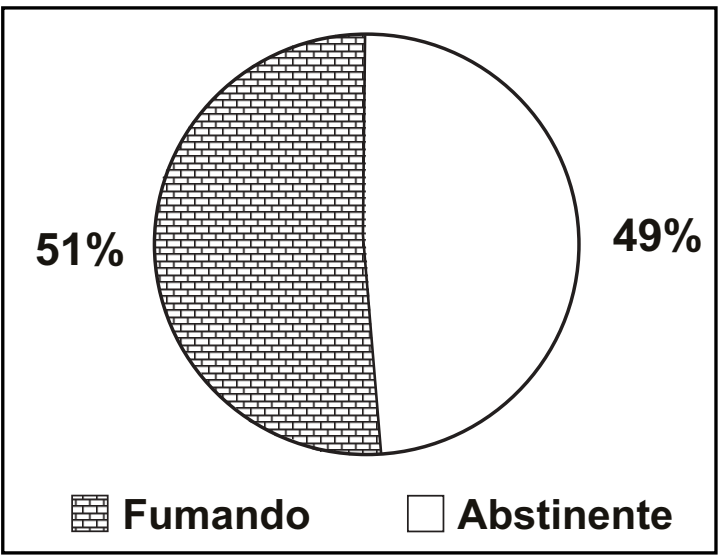

Figura 4. Porcentaje de abstinencia en el grupo que usó bupropión.

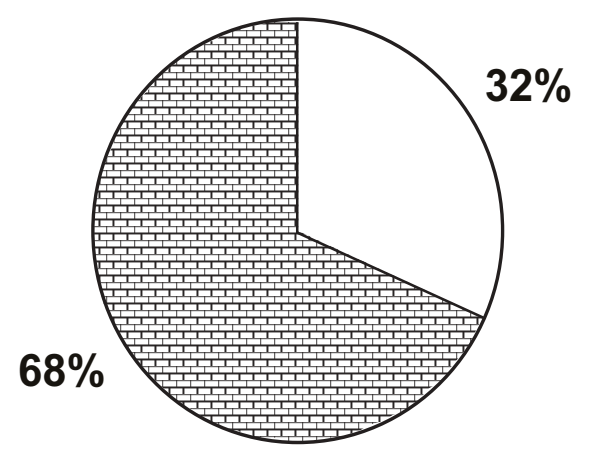

圈 Fumando

Abstinente

Figura 5. Porcentaje de abstinencia en el grupo que no usó bupropión.
Si bien la patología respiratoria como factor de riesgo no aparece como una variable significativa, presenta una probabilidad asociada cercana a 0,05 $(0,06)$; lo mismo ocurre con la variable género, cuya probabilidad asociada fue 0,07 (Tabla 2).

En la asociación entre consumo de tabaco a un año y todo el resto de variables en estudio no se encontró asociación estadísticamente significativa (Tabla 2).

\section{Discusión}

En esta muestra, la proporción de personas con alguna patología respiratoria, es mayor que en la población general $(33,82 \text { versus } 21,7)^{2}$, la presencia de estas enfermedades podrían ser una motivación para inscribirse en un programa antitabaco. Lo anterior no ocurre con la presencia de HTA, ya que en esta muestra se presentó una menor proporción de HTA que en la población general (27,94 versus $33,7)^{2}$, lo que podría hacer pensar, que las personas no consideran la presencia de HTA como un motivo para ingresar a un programa antitabaco, probablemente porque es generalmente asintomática.

El consumo diario de cigarrillos (previo al tratamiento) también presentó una diferencia con la población general (20 versus 8 cigarrillos diarios) $^{2}$, de esta diferencia de 14 cigarrillos, puede concluirse que a mayor consumo diario, mayor motivación para incluirse en un plan para dejar de fumar.

En relación a la salud mental, el antecedente de depresión se presenta en mayor proporción que la población general, lo cual es concordante con lo encontrado en el estudio de Seijas y col publicado en $1999^{6}$. El consumo de benzodiazepinas, en esta muestra, resultó ser mayor que la encontrada en un estudio realizado en una población consultante de atención primaria en Chile, en la que fue 4,8 puntos menor (30,88 versus 26$)^{9}$, todo esto se condice con la literatura existente, que muestra una asociación entre patología mental y consumo de tabaco ${ }^{10}$, esto es relevante, si se considera que los pacientes que tienen antecedentes de depresión presentan mayor adicción a la nicotina, lo que hace que tengan un mayor fracaso en este tipo de tratamientos ${ }^{11}$.

Según la literatura existente, los programas para dejar de fumar tienen una eficacia cercana a 
$30 \%$ y $35 \%$ a un año plazo, es por esto que $41,18 \%$ es un buen resultado. Sin embargo, se debe considerar que estos pacientes presentan alta motivación para dejar de fumar, ya que documentaron por adelantado el pago del programa, lo cual por un lado asegura la asistencia a las sesiones y, además, constituye un nuevo incentivo para dejar de fumar, pese a lo anterior, resulta útil considerar la metodología empleada en este estudio en la elección de un tratamiento antitabaco.

En el estudio descriptivo agrupado por género, sólo es significativa la asociación del género con el IMC, presentando un mayor IMC las mujeres que los hombres, diferencia que coincide con la encontrada en la encuesta nacional de salud, en el estrato socioeconómico alto ${ }^{2}$.

El bupropión como factor protector está avalado ampliamente por la literatura existente, así lo demuestran las guías de práctica clínica 2000 "Treating tobacco use and dependence"4 y la revisión Cochrane ${ }^{5}$. El uso de bupropión en este estudio resultó ser un factor protector de consumo de tabaco a un año de tratamiento, con un OR de 3,95 , en la guía de práctica clínica referida el OR obtenido fue $2,1^{5}$ y en la revisión Cochrane 1,97 $(95 \% \text { CI } 1,67-2,34)^{5}$, por tanto la asociación encontrada en este estudio fue más fuerte.

Los SN no demostraron ser efectivos, lo cual es distinto de lo encontrado en la guía de práctica clínica 2000 "Treating tobacco use and dependen$\mathrm{ce}^{\prime \prime 4}$ donde aparece como factor protector con un OR de 1,9; la revisión Cochrane donde el OR fue $1,74^{5}$ y el estudio de Seijas y col en una población de similares características ${ }^{6}$, esta divergencia pudiera ser porque $77,94 \%$ de la muestra usó los SN, lo cual pudiera enmascarar una real asociación. No se realizó en este estudio un análisis de la respuesta a tratamiento de grupos específicos, como el de aquellos que recibieron bupropión y sustitutos de nicotina, ya que se estudió cada una de las variables en forma independiente del resto.

Modified Reasons for Smoking Scale (MRSS), es la escala de motivación para fumar de la Universidad de Londres traducida al francés, que fue estudiada en Francia en 313 pacientes en 2003, encontrándose una relación estadísticamente significativa entre puntaje alto en el ítem automático $\mathrm{y}$ fracaso en dejar de fumar, y un puntaje significativamente mayor en mujeres, para los ítem sedación, estimulación e imagen psicológica ${ }^{12}$. Al respecto, en este estudio no se presentó una diferencia significativa en los puntajes de los ítems de motivación entre los hombres y las mujeres, y el ítem automático resultó ser un factor de riesgo importante al ajustar por las otras variables en estudio, con un OR de 9,3 (95\% IC 1,28-41,6), este resultado es coincidente con el estudio en Francia, puede decirse entonces, que es útil considerar al grupo de pacientes con un puntaje alto en el ítem automático, como aquel que va a requerir un apoyo mayor para dejar de fumar.

El género masculino aparece asociado a la variable respuesta en la regresión univariada, donde aparece como factor protector; en la regresión multivariada, si bien no alcanza significancia estadística, presenta un valor de $\mathrm{p}$ cercano a ésta $(0,07)$, lo que concuerda con la bibliografía existente ${ }^{13-15}$.

La presencia de patología respiratoria, aparece cercana a la significancia estadística como un factor de riesgo para el fracaso a tratamiento, resultado que es discordante con la literatura ${ }^{16}$.

Algunas variables que en la literatura aparecen significativas y que en este estudio no demostraron estar asociadas con el consumo de tabaco a un año, fueron: edad, consumo diario de cigarrillos, puntaje de Fagerstrom, presencia de tabáquicos en el hogar y presencia de HTA, todas estas variables pudieran alcanzar significancia estadística, al estudiarlas en una muestra de mayor tamaño y cubriendo diferentes niveles socioeconómicos ${ }^{13,15}$.

Entre las limitaciones de este estudio están la exclusión de los pacientes que no pudieron ser ubicados, lo que correspondió a $35,34 \%$ de la muestra; la indicación no aleatoria de bupropión y $\mathrm{SN}$, basada más bien en criterios médicos y en la aceptación por parte del paciente a recibirlo; y por último, el tamaño muestral de 68 que predispone a error aleatorio.

Pese a las limitaciones que este estudio presenta, los aportes que hace son: ser el primer estudio en Chile, que analiza la asociación de bupropión y SN con el cese de fumar. Además, describimos un grupo en el cual tendrá menor eficacia un tratamiento antitabaco: alto puntaje en el ítem automático de motivación para fumar. En este aspecto además, se da un primer acercamiento a nivel nacional, al estudio de determinantes en el cese de fumar luego de un tratamiento antitabaco, evaluando la motivación para fumar como determinante en el cese de consumo, sobre lo cual existe escasa bibliografía. 


\section{REFERENCIAS}

1. World Health Organization,World Health Report 1999 (Geneva: WHO,1999). www.who.int/whr/ 1999

2. Encuesta Nacional de Salud. Chile 2003. Biblioteca Virtual Ministerio de Salud. www.minsal.gov.cl.

3. Departamento de Estadísticas e Informaciones en Salud. Ministerio de Salud. Chile. 2002. www.minsal.gov.cl

4. Treating Tobacco Use and Dependence, Clinical Practice Guideline, 2000. www.ahrq.gov/path/ tobacco

5. Cochrane Database of Systematic Reviews. 2006, volume 3. www.theCochraneLibrary.com

6. Sejjas D, Santander J, Ferrer V, Ramos A. Dependencia de nicotina: seguimiento a un año plazo de pacientes tratados con terapia grupal más reemplazo de nicotina. Rev Méd Chile 1999; 127: 1313-20.

7. Sánchez E, Alvaro JL. Medición de la Salud Mental. Rev Chil Salud Pública 2002; 6: 41-51.

8. Heatherton T, Kozlowski L, Frecker R, Fagerstrom G. The Fagerstrom Test for Nicotine Dependence: a revision of the Fagerstrom Tolerance Questionnaire. Addiction 1991; 86: 1119-27.

9. Galieguilos T, Risco L, Garay JL, González M, Voguel M. Tendencia del uso de benzodiazepinas en una muestra de consultantes en atención primaria. Rev Méd Chile 2003; 131: 535-40.
10. Rojas G, Gaete J, González R, Ortega M, Figueroa A, Fritsch R, Araya R. Tabaquismo y Salud Mental. Rev Méd Chile 2003; 131: 873-80.

11. Anda RF, Wimiamson DF, Escobedo LG, Mast EE, Giovino GA, Remington PL Depression and the dynamics of smoking. A national perspective. JAMA 1990; 264: 1583-4.

12. Berlin I, Singleton EG, Pedarriosse AM, Lancrenon S, Rames A, Aubin HJ, Niaura R. The Modified Reasons for Smoking Scale: factorial structure, gender effects and relationship with nicotine dependence and smoking cessation in French smokers. Addiction 2003; 98: 1575-83.

13. Dale L, Glover E, Sachs D, Schroeder D, Offord K, Croghan I, Hurt R. Bupropion for Smoking Cessation Predictors of Successful Outcome. Prev Med 1994; 23: 335-44.

14. Hymowitz N, Cummings KM, Hyland A, Lynn WR, Pechacek TF, HaRtwell TD. Predictors of smoking cessation in a cohort of adult smokers followed for five years. Tob Control 1997; 6: 57-62.

15. Swan G, Jack L, Curry S, Chorost M, Javitz H, Mcafre T, DACEY S. Bupropion SR and counseling for smoking cessation in actual practice: Predictors of outcome. Nicotine and Tobacco Research 2003; 5: 911-21.

16. Nides MA, Rakos RF, Gonzales D, Murray RP, Tashin DP, BJornson-Benson WM et al. Predictors of initial smoking cessation and relapse through the first 2 years of the Lung Health Study. J Consult Clin Psychol 1995; 63: 60-9. 